\title{
Fruits and vegetables as a healthier snack throughout the day among families with older children: Findings from a survey of parent-child dyads
}

\author{
Authors: Teresa Smith, Courtney Pinard, Carmen Byker \\ Shanks, Holly Wethington, Heidi M. Blanck, Amy L. Yaroch
}

NOTICE: this is the author's version of a work that was accepted for publication in Eating Behaviors. Changes resulting from the publishing process, such as peer review, editing, corrections, structural formatting, and other quality control mechanisms may not be reflected in this document. Changes may have been made to this work since it was submitted for publication. A definitive version was subsequently published in Eating Behaviors, [VOL\# 17, April 2015] DOI\# https://dx.doi.org/10.1016/j.eatbeh.2015.01.006

Smith, Teresa M., Courtney A. Pinard, Carmen Byker Shanks, Holly Wethington, Heidi M. Blanck, and Amy L. Yaroch. "Fruits and vegetables as a healthier snack throughout the day among families with older children: Findings from a survey of parent-child dyads." Eating Behaviors 17 (April 2015): 136-139. DOI: https://dx.doi.org/10.1016/j.eatbeh.2015.01.006.

Made available through Montana State University's $\underline{\text { ScholarWorks }}$ scholarworks. montana.edu 


\title{
Fruits and vegetables as a healthier snack throughout the day among families with older children: Findings from a survey of parent-child dyads
}

\author{
Teresa M. Smith ${ }^{\mathrm{a}, \mathrm{d}, *}$, Courtney A. Pinard ${ }^{\mathrm{a}, \mathrm{d}}$, Carmen Byker Shanks ${ }^{\mathrm{b}}$, Holly Wethington ${ }^{\mathrm{c}}$, \\ Heidi M. Blanck ${ }^{c}$, Amy L. Yaroch ${ }^{\text {a,d }}$
}

\begin{abstract}
a Gretchen Swanson Center for Nutrition, 8401 West Dodge Road, Suite 100, Omaha, NE 68114, USA
${ }^{\mathrm{b}}$ Montana State University, Department of Health and Human Development, 222 Romney Gym, Bozeman, MT 59717, USA

${ }^{c}$ Division of Nutrition, Physical Activity, and Obesity, National Center for Chronic Disease Prevention \& Health Promotion, CDC, 1600 Clifton Rd. Atlanta, GA 30333, USA

d University of Nebraska Medical Center, Department of Health Promotion, Social \& Behavioral Health, UNMC College of Public Health, 984365 Nebraska Medical Center, Omaha, NE 68198-4365, USA
\end{abstract}

Keywords:

Snacking

Older children

Adolescents

Fruits and vegetables

ConsumerStyles

\begin{abstract}
Most U.S. youth fail to eat the recommended amount of fruits and vegetables (FV) however many consume too many calories as added sugars and solid fats, often as snacks. The aim of this study was to assess factors associated with serving FV as snacks and with meals using parent-child dyads. A cross-sectional sample of U.S. children aged 9 to 18 , and their caregiver/ parent $(n=1522)$ were part of a Consumer Panel of households for the 2008 YouthStyles mail survey. Chi-square test of independence and multivariable logistic regression were used to assess associations between serving patterns of FV as snacks with variations in serving patterns, and covariates including dietary habits. Most parents $(72 \%)$ reported serving FV at meals and as snacks. Fruit was most frequently served as a snack during the day (52\%) and vegetables were most frequently served as a snack during the day $(22 \%)$ but rarely in the morning. Significant differences in child FV intake existed among FV as a snack serving patterns by parents. Compared to children whose parents served FV only at meals, children whose parents reported serving FV as snacks in addition to meals were significantly more likely to have consumed FV the day before (using a previous day screener), $\mathrm{P}<0.05$. Contributing to the growing collection of literature describing parent-child dyad dietary behaviors, these findings suggest promoting FV access and intake throughout the day, not only at meals, by including serving as snacks, may increase FV intake among older children and adolescents.
\end{abstract}

\section{Introduction}

Older children and adolescents are consuming fruits and vegetables (FV) well below amounts recommended in the 2010 Dietary Guidelines for Americans (Centers for Disease Control \& Prevention, 2012; Dietary Guidelines Advisory Committee, 2010; Kimmons, Gillespie, Seymour, Serdula, \& Blanck, 2009) yet are consuming foods high in added sugars and solid fats above recommended amounts (Reedy \& Krebs-Smith, 2010), often as snacks (Slining \& Popkin, 2013). The 2010 National Youth Physical Activity and Nutrition Study showed that $28.5 \%$ of high school students consumed fruit less than once daily and $33.2 \%$ con-sumed vegetables less than once daily (Kim, Grimm, Harris, Scanlon, \& Demissie, 2011). Since the 1970s, fruit as a snack has decreased among children (aged 2-18), while snacks consisting of foods high in added sugars and solid fats have increased contributing to an intake of about $168 \mathrm{cal}$ more per day from snacking (Piernas \& Popkin, 2010).

Key settings for promoting healthy snacking for older children include schools, food stores, and the home food environment (Borradaile et al.,
2009; Story, Kaphingst, Robinson-O'Brien, \& Glanz, 2008). The home food environment has been characterized by several factors, including availability of FV, accessibility of FV, and parental intake of FV, which are consistently and positively related to FV intake in children and adoles-cents (Bere \& Klepp, 2004; Hearn et al., 1998; Jago, Baranowski, Baranowski, et al., 2007). There is scant information available however re-garding the relationship of serving FV as snacks and family FV intake habits. As the ubiquity of energy-dense snack foods among community retail venues (Farley, Baker, Futrell, \& Rice, 2010) and vending machines in school settings (Kubik, Lytle, \& Farbakhsh, 2011) has become a recent focus for intervention, focusing on serving FV as snacks by parents is one strategy to consider to improve diet quality among children and po-tentially reduce snacking on foods high in added sugars and solid fats.

This study used 2008 Styles data to address two objectives. The first was to describe the provision of FV as snacks and to identify significant differences among serving patterns. The second was to examine the 
relationship between parental provision of FV as snacks among older child/adolescents (aged 9-18) and FV intake. We hypothesized that children of parents who serve FV as a snack in addition to meals are more likely to consume FV than children of parents who only serve FV at meals.

\section{Materials and methods}

\subsection{Study sample}

Data from the Porter Novelli 2008 Styles (ConsumerStyles, HealthStyles, and YouthStyles) opinion panel surveys, administered by Synovate, Inc., were used for this study. ConsumerStyles participants were sampled from a consumer mail panel of approximately 340,000 households using a sampling design stratified by region, household income, population density, age, and household size, and included an oversample of low income/minority participants and householdswith-children to ensure adequate representation of these groups ( $n=20,000$ ). The response rate for ConsumerStyles was $51 \%$. HealthStyles was sent to adults ( $\geq 18$ years; $n=7000$ ) and YouthStyles was sent to youth (9-18 years; $n=2605$ ) whose household had returned ConsumerStyles. Response rates for 2008 HealthStyles and YouthStyles were $77 \%$ and $65 \%$, respectively.

Parent-reported variables were linked to YouthStyles data using a unique household identifier to create 1704 parent-child dyads. Respondents were excluded from analysis if the parent reported not serving FV in their home $(n=43)$, serving FV only as snacks and not at meals ( $n=20$ ), or if data for these questions were missing or incomplete ( $n=119$ ). Throughout this paper, "parent" refers to the parent or primary caregiver of the child. The final analytic sample consisted of 1522 parent-child dyads.

\subsection{Measures}

Two questions regarding provision of FV to one's family were included in the 2008 HealthStyles survey. The first question asked, "In general, when do you typically provide or serve fruit or $100 \%$ fruit juice to your family?" Response options included breakfast (100\% juice), breakfast (fruit), lunch or lunchbox, dinner, dessert, snack in the morning, snack during the day, or snack at night. The second question asked, "In general, when do you typically provide or serve vegetables to your family?" Response options included breakfast (juice or vegetable), lunch or lunchbox, dinner, snack in the morning, snack during the day, or snack at night. Parents were instructed to select all that apply. Parents reported their sex, age, education, race/ethnicity, marital status, and household income.

In YouthStyles, respondents were asked about their FV intake, which was assessed through a prior day screener. The question asked, "Now think about what you ate yesterday. Yesterday, how many times did you:" For this analysis, "eat fruit" and "eat vegetables" were combined into one item and analyzed; and responses were categorized in the following way: did not eat FV (0 times), 1 time, 2 times, 3 times, or 4 or more times. Child sex, age, height, and weight were collected from parents as part of the consumer panel data. Child body mass index (BMI)-for-age was calculated using percentiles based on the CDC growth reference year 2000 .

\subsection{Statistical analysis}

SAS (version 9.2 SAS Institute Inc., Cary, NC) was used for the statistical analyses. Analyses for this sample were post-stratified so that the weighted sample distribution of adults in terms of sex, age, income, race, and household size matched U.S. 2007 Current Population Survey proportions. Descriptive analyses examined parent reported serving patterns of FV as snacks. Chi-square tests of independence were used to assess relationships among child reported FV intake and parent reported serving patterns of FV as snacks; statistical significance was set at a two-sided alpha level of $P<0.05$. Multivariable logistic regression analyses were performed to examine whether FV serving patterns were associated with child consumption of FV adjusting for covariates. FV serving pattern responses were dichotomized into those who reported serving FV only at meals (breakfast, lunch or lunchbox, dinner, or dessert) versus those who reported serving FV at meals and as snacks (meal options and snack in the morning, snack during the day, or snack at night). Weighted percentages were analyzed with consideration of the complex survey sample design by applying Surveyfreq and Surveylogistic procedures. Adjusted odds ratios are reported with accompanying $95 \%$ confidence intervals.

\section{Results}

Of the weighted analytic sample of children, $48 \%$ were male, the mean age was 13.4 years ( $\mathrm{SE}=0.1$ ), and $38 \%$ were overweight or obese ( $\geq 85$ th percentile BMI-for-age; data not shown). Of the weighted analytic sample of parents, $33 \%$ were male; $56 \%$ were aged 18-54 years; $71 \%$ were non-Hispanic white; $46 \%$ had a household income less than $\$ 40,000$ annually; and $33 \%$ had completed college or more (data not shown). Most parents (72\%) reported serving FV at meals and as snacks. Results from a weighted chi-square analysis revealed that child's sex, age or body weight status, and parent's sex, race and/or ethnicity, household income, or education did not significantly differ between those who reported serving FV at meals and as snacks compared to those who reported serving FV only at meals (data not shown).

When considering all times throughout the day that parents served FV at a meal, they most frequently reported serving fruit at breakfast (100\% juice or fruit; $63 \%$ ), followed with lunch or in a lunchbox (44\%), and with dinner or dessert ( $38 \%$; data not shown). They most frequently reported serving vegetables most frequently at dinner (98\%), followed with lunch or in a lunchbox (39\%), and at breakfast (juice or vegetable; $38 \%$ ). When considering the parents reported patterns of serving FV as snacks, fruit was most frequently served during the day (52\%) and vegetables were most frequently served during the day (22\%) but rarely in the morning (4\%). Children in this study were significantly more likely to report intake of FV when their parent reported serving vegetables as a snack in the morning, during the day, or at night (Table 1). The number of times the child reported eating FV yesterday did not differ significantly based on serving patterns of fruit as a snack.

Results from the adjusted multivariable logistic regression model found that among children whose parent reported serving FV at meals and snacks were more likely to have consumed FV one time in the previous day compared to those who reported serving FV only at meals ( $\mathrm{OR}=1.69,95 \% \mathrm{CI}=1.14,2.51$; Table 2$)$. Children were also more likely to have consumed FV twice ( $\mathrm{OR}=1.55,95 \% \mathrm{CI}=1.05,2.29)$ or 4 or more times $(\mathrm{OR}=1.71,95 \% \mathrm{CI}=1.16,2.52)$ than to have not consumed FV yesterday when their parents reported serving FV at meals and snacks.

\section{Discussion}

The majority of parents in this study served FV at meals and snacks to their family, an association, which to our knowledge has not been reported previously with population-level data. However, we found differences in patterns when FV were served as snacks, revealing several opportunities for interventions. Less than half of parents reported serving fruits as a snack in the morning or at night; and less than half reported serving vegetables as a snack at any point throughout the day. Considering that children were significantly more likely to have reported consuming FV in the previous day when vegetables were served as a snack in the morning, during the day, or at night, new or ongoing initiatives can aim to specifically promote vegetables as a snack throughout the day to promote overall FV intake. However, each family 
Serving patterns of fruits and vegetables as snacks by parents and the self-reported FV intake of their children, aged 9-18 ( $n=1522$ ).

\begin{tabular}{|c|c|c|c|c|c|c|c|c|c|c|c|c|}
\hline & \multicolumn{6}{|c|}{ Fruits provided as a snack by parent } & \multicolumn{6}{|c|}{ Vegetables provided as a snack by parent } \\
\hline & \multicolumn{2}{|c|}{ In the morning } & \multicolumn{2}{|c|}{ During the day } & \multicolumn{2}{|l|}{ At night } & \multicolumn{2}{|c|}{ In the morning } & \multicolumn{2}{|c|}{ During the day } & \multicolumn{2}{|l|}{ At night } \\
\hline & $\%(\mathrm{SE})$ & $P$ value & $\%(\mathrm{SE})$ & $P$ value & $\%(\mathrm{SE})$ & $P$ value & $\%(\mathrm{SE})$ & $P$ value & $\%(\mathrm{SE})$ & $P$ value & $\%(\mathrm{SE})$ & $P$ value \\
\hline Total & $21.23(1.12)$ & - & $51.63(1.39)$ & - & $34.74(1.31)$ & - & $3.94(0.55)$ & - & $22.29(1.13)$ & - & $12.92(0.94)$ & - \\
\hline \multicolumn{13}{|c|}{$\begin{array}{l}\text { Number of times FV eaten } \\
\text { by child yesterday }\end{array}$} \\
\hline Did not eat FV & $12.81(1.93)$ & 0.260 & $13.41(1.28)$ & 0.135 & $12.66(1.52)$ & 0.152 & $7.45(3.82)$ & $0.049^{*}$ & $13.14(1.90)$ & $0.010^{*}$ & $9.88(2.38)$ & $0.035^{*}$ \\
\hline 1 time & $24.66(2.73)$ & & $23.40(1.66)$ & & $22.32(2.02)$ & & $36.56(7.48)$ & & $27.76(2.75)$ & & $28.32(3.85)$ & \\
\hline 2 times & $22.27(2.48)$ & & $26.51(1.75)$ & & $24.90(2.06)$ & & $29.29(6.39)$ & & $27.54(2.55)$ & & $29.50(3.65)$ & \\
\hline 3 times & $13.60(2.09)$ & & $13.12(1.27)$ & & $12.85(1.55)$ & & $8.52(3.70)$ & & $10.85(1.75)$ & & $10.78(2.35)$ & \\
\hline 4 or more times & $26.67(2.67)$ & & $27.28(2.08)$ & & $27.28(2.08)$ & & $18.18(5.53)$ & & $20.70(2.26)$ & & $21.52(3.27)$ & \\
\hline
\end{tabular}

* Denotes significant associations at $P<0.05$ (two-tailed).

a Unweighted frequencies and weighted percentages reported.

may have different behavioral practices and preferences that may need to be taken into account when interacting with them.

Children of families in which the parent reported serving FV as snacks in addition to meals reported greater FV intake on the previous day when compared to those who serve FV only at meals. These findings suggest that provision of FV as snacks could potentially increase overall FV intake among children. While it may seem most impactful to simply promote general FV provision in the home, research has shown that among elementary school children, FV are most frequently consumed during weekday lunch and dinner and few FV are consumed as a snack, supporting recommendations for interventions to increase FV intake as snacks (Baranowski et al., 1997; Lazzeri et al., 2013). This study shows that when parents provide FV as snacks there may be higher consumption among older children. Interventions designed to influence child or adolescent FV intake by promoting fruits, and especially vegetables, as snacks should target parents and consider incorporating strategies to reach families in their homes (Gross, Pollock, \& Braun, 2010; Robinson-O'Brien, Neumark-Sztainer, Hannan, Burgess-Champoux, \& Haines, 2009).

Limitations in the current study included using self-reported data, potentially introducing biases such as recall. Because cross-sectional survey data was used, causality cannot be determined. Also, participants were not randomly selected, as they came from a panel survey and the response rate for the 2008 ConsumerStyles survey suggests a low response. Finally, a previous day screener was used to assess FV intake among children, so it does not reflect usual intake or allow for estimation of nutrient intake. Despite these limitations, the findings add to the evidence base to augment understanding of factors related to FV intake by increasing provision of FV by parents or in the home.
Strengths are that the data were collected from a relatively large population-based sample, with oversampling in low-income individuals and minorities to increase representation of these groups.

\section{Conclusion}

This study was unique in that, to our knowledge, it was the first to explore behaviors of parent-child dyads where FV are served as snacks in addition to meals. Contributing to the growing body of parent-child dyad literature on dietary behaviors, these findings highlight data that promoting FV access and intake throughout the day, including serving fruits and vegetables as snacks may benefit FV intake among older children and adolescents.

\section{Role of funding sources}

No financial support was provided for the conduct of the research and/or preparation of the manuscript.

\section{Contributors}

All authors contributed to the conception of this work. T.M.S. analyzed the data and wrote the first draft of the manuscript. C.A.P., C.J.B., H.W., H.M.B., and A.L.Y. provided extensive guidance throughout the study and writing of the manuscript. All authors contributed to and have approved the final manuscript.

\section{Conflict of interest}

There are no conflicts of interest by any author.

Table 2

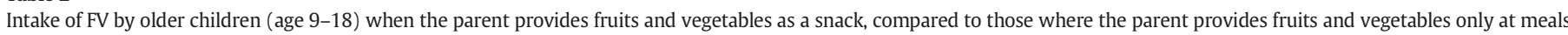
(logistic regression, controlling for demographic characteristics) $(n=1522){ }^{\mathrm{a}}$

\begin{tabular}{|c|c|c|c|c|c|}
\hline & \multicolumn{2}{|c|}{ Total } & \multirow{2}{*}{$\frac{\text { Provide FV only at meals }}{\%(\mathrm{SE})}$} & \multirow{2}{*}{$\frac{\text { Provide FV at meals and as a snack }}{\%(\mathrm{SE})}$} & \multirow[t]{2}{*}{ Adjusted OR (95\% CI) } \\
\hline & $\mathrm{n}$ & $\%(\mathrm{SE})$ & & & \\
\hline \multicolumn{6}{|c|}{ Number of times FV eaten by child yesterday } \\
\hline Did not eat FV & 231 & $15.39(0.99)$ & $5.47(0.63)$ & $9.92(0.81)$ & 1.00 \\
\hline 1 time & 317 & $21.51(1.15)$ & $5.03(0.59)$ & $16.48(1.05)$ & $1.69(1.14,2.51)^{* *}$ \\
\hline 2 times & 355 & $25.27(1.29)$ & $7.04(0.84)$ & $18.23(1.11)$ & $1.55(1.05,2.29)^{*}$ \\
\hline 3 times & 203 & $13.71(0.95)$ & $4.82(0.59)$ & $8.89(0.78)$ & $1.05(0.68,1.61)$ \\
\hline 4 or more times & 350 & $24.12(1.23)$ & $6.19(0.78)$ & $17.94(1.06)$ & $1.71(1.16,2.52)^{* *}$ \\
\hline
\end{tabular}

Note: $\mathrm{OR}=$ odds ratio; $\mathrm{CI}=$ confidence interval; Reference category is "Provide FV at meals and as snack".

a Unweighted frequencies and weighted percentages reported.

b Model adjusts for parental sex, age, race and/or ethnicity, household income, education, and marital status.

* $P<0.05$.

** $P<0.01$. 


\section{References}

Baranowski, T., Smith, M., Hearn, M.D., Lin, L.S., Baranowski, J., Doyle, C., et al. (1997). Patterns in children's fruit and vegetable consumption by meal and day of the week. Journal of the American College of Nutrition, 16(3), 216-223.

Bere, E., \& Klepp, K.I. (2004). Correlates of fruit and vegetable intake among Norwegian schoolchildren: Parental and self-reports. Public Health Nutrition, 7, 991-998.

Borradaile, K.E., Sherman, S., Vander Veur, S.S., McCoy, T., Sandoval, B., Nachmani, J., et al. (2009). Snacking in children: The role of urban corner stores. Pediatrics, 124(5), 1293-1298.

Centers for Disease Control and Prevention (2012). Adolescent and School Health. Retrieved July 16, 2012, from http://www.cdc.gov/healthyyouth/nutrition/facts.htm

Dietary Guidelines Advisory Committee (2010). Report of the Dietary Guidelines Advisory Committee on the Dietary Guidelines for Americans, 2010, to the Secretary of Agriculture and the Secretary of Health and Human Services. Agricultural Research Service.

Farley, T.A., Baker, E.T., Futrell, L., \& Rice, J.C. (2010). The ubiquity of energy-dense snack foods: A national multicity study. American Journal of Public Health, 100(2), 306-311.

Gross, S.M., Pollock, E.D., \& Braun, B. (2010). Family influence: Key to fruit and vegetable consumption among fourth-and fifth-grade students. Journal of Nutrition Education and Behavior, 42(4), 235-241.

Hearn, M.D., Baranowski, T., Baranowski, J., Doyle, C., Smith, M., Lin, L.S., et al. (1998). Environmental influences on dietary behavior among children: Availability and accessibility of fruits and vegetables enable consumption. Journal of Health Education, 29(1), 26-32.

Jago, R., Baranowski, T., \& Baranowski, J.C. (2007). Fruit and vegetable availability: A micro environmental mediating variable? Public Health Nutrition, 10(7), 681-689.

Kim, S.A., Grimm, K.A., Harris, D.M., Scanlon, K.S., \& Demissie, Z. (2011). Fruit and vegetable consumption among high school students. Morbidity and Mortality Weekly Report, 60(46), 1583-1586.
Kimmons, J., Gillespie, C., Seymour, J., Serdula, M., \& Blanck, H.M. (2009). Fruit and vegetable intake among adolescents and adults in the United States: Percentage meeting individualized recommendations. The Medscape Journal of Medicine, 11(1), 26.

Kubik, M.Y., Lytle, L.A., \& Farbakhsh, K. (2011). School and district wellness councils and availability of low-nutrient, energy-dense vending fare in Minnesota middle and high schools. Journal of the American Dietetic Association, 111(1), 150-155.

Lazzeri, G, Pammolli, A Azzolini, E Simi, R, Meoni, V, de Wet D, et al (2013). Association between fruits and vegetables intake and frequency of breakfast and snacks consumption: A cross-sectional study. Nutrition Journal, 12(1), 123.

Piernas, C., \& Popkin, B.M. (2010). Trends in snacking among U.S. children. Health Affairs (Project Hope), 29(3), 398-404. http://dx.doi.org/10.1377/hlthaff.2009.0666.

Reedy, J., \& Krebs-Smith, S.M. (2010). Dietary sources of energy, solid fats, and added sugars among children and adolescents in the United States. Journal of the American Dietetic Association, 110(10), 1477-1484.

Robinson-O'Brien, R., Neumark-Sztainer, D., Hannan, P.J., Burgess-Champoux, T., \& Haines, J. (2009). Fruits and vegetables at home: Child and parent perceptions. Journal of Nutrition Education and Behavior, 41(5), 360-364. http://dx.doi.org/10.1016/j.jneb. 2008.08.003.

Slining, M.M., \& Popkin, B.M. (2013). Trends in intakes and sources of solid fats and added sugars among U.S. children and adolescents: 1994-2010. Pediatric Obesity, 8(4), 307-324. http://dx.doi.org/10.1111/j.2047-6310.2013.00156.x.

Story, M., Kaphingst, K.M., Robinson-O'Brien, R., \& Glanz, K. (2008). Creating healthy food and eating environments: Policy and environmental approaches. Annual Review of Public Health, 29, 253-272. http://dx.doi.org/10.1146/annurev. publhealth.29.020907.090926. 